\title{
Papillary thyroid carcinoma risk factors in the Yunnan plateau of southwestern China
}

\author{
This article was published in the following Dove Press journal: \\ Therapeutics and Clinical Risk Management \\ 30 June 2016 \\ Number of times this article has been viewed
}

\author{
Rong Zeng ${ }^{1-3}$ \\ Tao Shou ${ }^{3}$ \\ Kun-xian Yang ${ }^{4}$ \\ Tao Shen ${ }^{5}$ \\ Jin-ping Zhang ${ }^{5}$ \\ Rong-xia Zuo ${ }^{5}$ \\ Yong-qing Zheng ${ }^{5}$ \\ Xin-ming Yan $^{5}$
}

'Faculty of Environmental Science and Engineering, Kunming University of Science and Technology, Kunming, People's Republic of China; ${ }^{2}$ Faculty of Life Science and Technology, Kunming University of Science and Technology, Kunming, People's Republic of China; 'Medical Oncology, The First People's Hospital of Yunnan Province, Kunming, People's Republic of China; ${ }^{4}$ Surgical Oncology, The First People's Hospital of Yunnan Province Kunming, People's Republic of China; Institute of Clinical and Basic Medicine Research, The Affiliated Hospital of Kunming University of Science and Technology, Kunming, People's Republic of China

Correspondence: Xin-ming Yan Institute of Clinical and Basic Medicine Research, The Affiliated Hospital of Kunming University of Science and Technology, Kunming 650032, People's Republic of China Emailyxmin08@I63.com
Objective: This study investigated clinical and pathological characteristics and risk factors in papillary thyroid carcinoma (PTC) patients' native to Yunnan plateau in southwestern China. Methods: Clinical data from 1,198 patients diagnosed with PTC $(n=578)$ and control subjects ( $n=620$ ) with benign thyroid disease (ie, thyroid nodule disease, benign thyroid diseases [BTD]) in Yunnan province were analyzed retrospectively.

Results: The mean patient age was lower for PTC than for BTD. Positive ratios of thyroid peroxidase antibody, thyroglobulin antibody (TGAb), and thyrotrophin receptor antibody (TRAb) were higher in PTC than in BTD patients. The ratio of PTC coexisting with Hashimoto's thyroiditis (HT) or with lymphocytic thyroiditis was higher than that of BTD. The number of patients whose age at menarche was $\leq 13$ years, who had given birth to less than or equal to two children, or who were in premenopause were higher in the PTC than in the BTD group. Multivariate conditional logistic regression analyses revealed that age $>45$ years, nodal size $>1 \mathrm{~cm}$, and elevated TG levels were protective factors against PTC. Abnormally elevated TGAb and TRAb levels were independent risk factors for PTC in females.

Conclusion: HT was not an independent risk factor for but was associated with PTC. TRAb is a risk factor for PTC in individuals living in the Yunnan plateau, but not for those in the plains region.

Keywords: papillary thyroid carcinoma, risk factors, Yunnan plateau, benign thyroid disease, autoimmune disease status

\section{Introduction}

Thyroid cancer affects endocrine organs and has one of the highest incidence rates among thyroid diseases, which have markedly increased in the recent years. ${ }^{1}$ Indeed, thyroid cancer has become the fastest growing type of solid malignancy; ${ }^{2,3}$ from 1975 to 2006, the incidence in the US increased 2.6-fold, and it now ranks as the fifth most common malignancy among females. ${ }^{4}$

Papillary thyroid carcinoma (PTC) is the most common subtype of thyroid cancer, accounting for approximately $60 \%-80 \%$ of cases in adults and children, and is characterized by a high degree of differentiation and lower malignancy. ${ }^{4}$ Nonetheless, most of the epidemiological surveys have shown that it is the fastest growing subtype of thyroid cancer that encompasses follicular cancer, medullary carcinoma, and undifferentiated carcinoma. A variety of factors are associated with the development of thyroid cancer, including ionizing radiation, defects in iodine uptake, autoimmune thyroid disease, levels of thyroid-stimulating hormone (TSH) and its receptor, estrogen and progestin levels, and body mass index (BMI) as well as genetic, social, and cultural factors. ${ }^{5}$ Thyroid cancer often coexists with other benign thyroid diseases (BTDs) such as 
nodular goiter, Hashimoto's thyroiditis (HT), chronic lymphocytic thyroiditis (LT), thyroid adenoma, and Graves' disease (GD). ${ }^{6}$

The incidence of thyroid cancer in People's Republic of China has also risen in the recent years; the annual incidence increased by $14.51 \%$ among females between 2003 and 2007. ${ }^{7}$ In Tianjin, People's Republic of China, the thyroid cancer incidence among females increased from 1.3 per 100,000 in 1981 to 4.2 per 100,000 in $2001 ; ;^{8}$ in Beijing, 862 cases of thyroid cancer were reported in 2006-2007 as compared to 258 cases in 1998-1999, making it one of the fastest growing cancer types. ${ }^{9}$ Similar trends have been observed in Shanghai and Hong Kong: the age-standardized incidence of thyroid cancer increased by an average of 3.1\% among men and 3.8\% among women per year in Shanghai from 1973 to 2009 , and by $2.2 \%$ and $2.7 \%$, respectively, in Hong Kong from 1983 to $2011 .{ }^{10}$ Meanwhile, the mean age of thyroid cancer patients is decreasing. ${ }^{11}$

Geographical factors also affect the development of cancer. One study carried out by the US Air Force, Navy, and Armed Forces Institute for pilots showed that exposure to high altitudes and/or aviator status was correlated with the incidence of skin, testicular, bladder, and thyroid cancers. ${ }^{12}$ A recent study revealed space-time differences in thyroid carcinoma mortality in Italy. The study also established a link between iron deficiency, residence in mountainous areas, and the mortality rate of thyroid carcinoma. ${ }^{13}$

Yunnan Province is located in People's Republic of China's Yunnan-Guizhou plateau, where the watershed of the Yangtze River meets the Pearl River Highlands. The altitude of Yunnan province is 1,500-2,000 $\mathrm{m}$ above sea level, with some of the mountain peaks reaching heights of $>3,000 \mathrm{~m}$. Yunnan is also a multiethnic enclave comprising a total of 26 ethnic groups in a population of 46 million, including a Han majority $(69.6 \%)$ as well as Yi (11\%), Hani (3.5\%), Bai (3.4\%), Dai (2.6\%), Zhuang (2.6\%), and other ethnicities, according to the 2011 national census. Various studies have investigated the prevalence of and factors associated with PTC in People's Republic of China and other countries, but the population living at moderate altitudes $(1,500-2,500 \mathrm{~m})$ on the Yunnan-Kweichow plateau in southwestern China has not been included in any surveys to date. So, this present study investigated risk factors associated with PTC in this subpopulation.

\section{Methods}

\section{Patients}

Patients with PTC and BTD $(n=1,198)$ were recruited from Yunnan First People's Hospital from January 2003 to December 2012. All patients were informed about the study, and a written consent was obtained to use their clinical data. Clinical data and pathological subtype were assessed using a case-control approach. Patients fulfilled the following criteria: 1) not taking L-T4 or methimazole and not exhibiting overt hyper-/hypothyroidism; 2) having undergone TSH, free thyroid hormones, and thyroglobulin antibodies (TGAbs) measurements within 1 week prior to thyroidectomy; and 3) not having previously undergone thyroid surgery of any type. Patients exhibiting diffuse lymphocytic and plasma cell infiltration, oxyphilic cells, and lymphoid follicles with reactive germinal centers were defined as having HT; a diagnosis of LT was made in the absence of oxyphilic cells.

\section{Data collection}

Information on clinical presentation as well as age, sex, marital status, fertility state, and presence of BTDs were recorded. Thyroid function tests measuring TSH (normal value: $0.3-5.5 \mathrm{IU} / \mathrm{mL}$ ) and antithyroid antibody levels - that is, against thyroid peroxidase antibody (TPOAb), TGAb, and thyrotrophin receptor antibody (TRAb) - were recorded. $\mathrm{TGAb}>30 \%$, TPOAb $>20 \%$, and TRAb $>5$ U/L were considered positive. TSH and antithyroid antibody levels were assessed in our hospital laboratory using radioimmunoassay kits (Tianjin Xiehe Medical Science Co., Ltd., Tianjin, People's Republic of China) and a DFM-96 Geiger counter (Zhongchen Mechanical and Electrical Co. Ltd, Foshan, People's Republic of China).

\section{Statistical analysis}

Data were analyzed using SPSS v.17.0 (SPSS Inc., Chicago, IL, USA) and reported as mean \pm standard deviation. Differences in thyroid cancer characteristics between groups were assessed with the $\chi^{2}$ test. A univariate analysis was used to screen factors related to thyroid cancer, and a multivariate logistic regression analysis was used to evaluate PTC risk. In all cases, $P<0.05$ was considered statistically significant. All data were analyzed anonymously. The study was approved by the Institutional Review Board of Yunnan First People's Hospital.

\section{Results}

\section{Clinical characteristics}

This study examined 1,198 patients aged between 9 and 86 years. The PTC group comprised 578 cases with a mean age of $42.35 \pm 13.46$ years, while 620 patients had BTD with a mean age of 50.11 12.94 years. The average age of the PTC group was lower than that of the BTD group $(P<0.001)$. Nodule diameter was smaller in the PTC than in the BTD group $(2.19 \pm 1.35$ vs $2.88 \pm 1.24 \mathrm{~cm})(P<0.001)$. Follicular adenoma was the most common type of benign 
Table I Distribution of main pathological types and their percentage

\begin{tabular}{lll}
\hline Pathological type & Numbers of patients & Percentage \\
\hline PTC & 578 & \\
FVPTC & 39 & 6.7 \\
BTD & 620 & \\
Follicular adenoma & 291 & 46.9 \\
Nodular goiter & 244 & 39.4 \\
Cystic thyroid adenoma & 59 & 9.5 \\
Acidophil thyroid neoplasia & 15 & 2.5 \\
Atypical thyroid adenoma & II & 1.7 \\
\hline
\end{tabular}

Abbreviations: BTD, benign thyroid disease; FVPTC, follicular variant of papillary thyroid carcinomas; PTC, papillary thyroid carcinoma.

nodule (46.9\%; Table 1). A high incidence of PTC was observed among patients between 35 and 44 years of age, who accounted for $29.8 \%$ of total cases; this age group also had the second highest incidence of benign thyroid nodules (Figure 1), a diagnosis that was confirmed either through reevaluation of histological sections (when available) or by reviewing previous pathology reports. The histological type of thyroid cancer was determined by two senior pathologists according to World Health Organization (WHO) criteria; among PTC patients, 39 had a follicular variant of PTC (6.7\%); among BTD patients, 291 (46.9\%) had follicular adenoma, 244 (39.4\%) had nodular goiter, 59 (9.5\%) had cystic thyroid adenoma, 15 (2.5\%) had acidophil thyroid neoplasia, and $11(1.7 \%)$ had atypical thyroid adenoma.

\section{Sex}

Age at PTC diagnosis was higher in males than in females (45.20 \pm 13.64 vs $41.81 \pm 13.38$ years; $P=0.027$ ). Lymph node metastasis was more prevalent among men than women (45.7\% vs $32.9 \% ; P=0.019)$. The proportion of cases in which PTC coexisted with HT or LT was higher for women than for men ( $13.4 \%$ vs $5.4 \%$ for HT and $4.3 \%$ vs $0.0 \%$ for

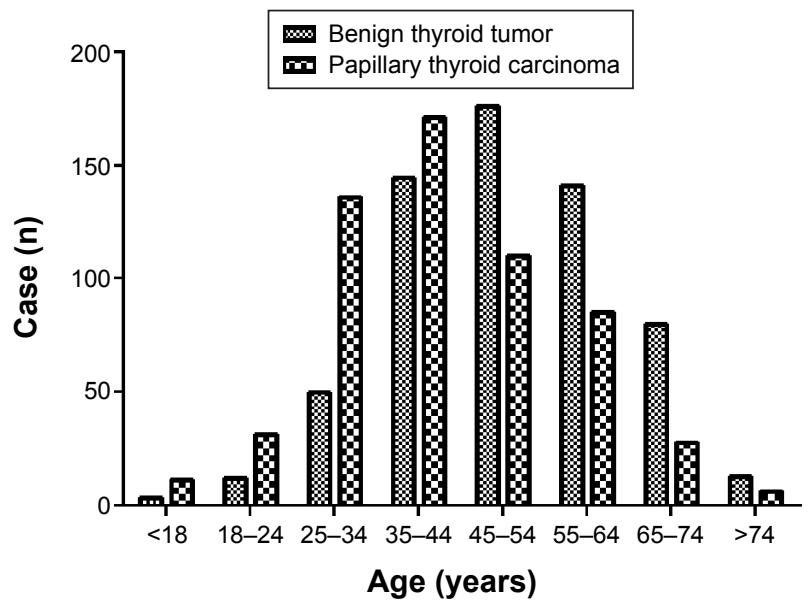

Figure I Distribution of patients with thyroid nodules by age.
LT; $P=0.032$ and 0.035 , respectively). There were no differences between the sexes in terms of tumor size, tumornode-metastasis (TNM) staging, or coexistence with nodular goiter or adenoma (Table 2).

\section{Univariate analysis}

\section{Preoperative serum TSH level}

Preoperative serum TSH concentration was higher in PTC than in BTD patients $(2.78 \pm 4.16$ vs $2.27 \pm 2.84 \mathrm{mU} / \mathrm{L}$; $P=0.018$ ), and more number of PTC patients had elevated preoperative serum TSH concentration $(7.5 \%$ vs $3.4 \%$; $P=0.003$ ) (Table 3).

\section{Thyroid autoimmune antibody levels}

There were 89 cases (19.1\%) that were TGAb-positive, 90 (20.4\%) that were TPOAb-positive, and 117 (31.0\%) that were TRAb-positive in the PTC group, which was significantly higher than the percentages observed in the BTD group $(P<0.001$; Table 3$)$, suggesting that thyroid autoimmune antibodies are biomarkers of PTC.

\section{HT and LT}

There were 70 cases of PTC (12.1\%) coexisting with HT, as compared to 35 BTD cases $(5.6 \%)(P<0.001)$. Similarly, the number of cases of PTC coexisting with LT $(21 ; 3.6 \%)$ was higher than BTD cases with coexisting LT $(9 ; 1.5 \%)$ $(P=0.02)$ (Table 3$)$. After excluding PTC cases coexisting

Table 2 Association between PTC and sex

\begin{tabular}{|c|c|c|c|}
\hline & Male $(n=92)$ & Female $(n=486)$ & $P$-value \\
\hline Age (years) & $45.20 \pm 13.64$ & $41.81 \pm 13.38$ & 0.027 \\
\hline Tumor size $(\mathrm{cm})$ & $2.22 \pm 1.20$ & $2.19 \pm 1.38$ & $0.84 I$ \\
\hline \multicolumn{4}{|l|}{ TNM staging (\%) } \\
\hline I & $65(72.2)$ & $371(77.8)$ & \multirow[t]{4}{*}{0.321} \\
\hline II & $9(10.0)$ & $37(7.8)$ & \\
\hline III & $9(10.0)$ & $22(4.6)$ & \\
\hline IV & $7(8.5)$ & 47 (9.9) & \\
\hline \multicolumn{4}{|c|}{ Lymphatic metastasis (\%) } \\
\hline With & $42(45.7)$ & $160(32.9)$ & \multirow[t]{2}{*}{0.019} \\
\hline Without & $50(54.3)$ & $326(67.1)$ & \\
\hline \multicolumn{4}{|c|}{ Nodular goiter (\%) } \\
\hline With & $14(15.2)$ & $64(13.2)$ & \multirow[t]{2}{*}{0.598} \\
\hline Without & $78(84.8)$ & $436(86.8)$ & \\
\hline \multicolumn{4}{|c|}{ Follicular adenoma (\%) } \\
\hline With & $20(21.7)$ & $88(18.1)$ & \multirow[t]{2}{*}{0.412} \\
\hline Without & $72(78.3)$ & $398(81.9)$ & \\
\hline \multicolumn{4}{|l|}{ HT (\%) } \\
\hline With & $5(5.4)$ & $65(13.4)$ & \multirow[t]{2}{*}{0.032} \\
\hline Without & $87(94.6)$ & $421(86.6)$ & \\
\hline \multicolumn{4}{|l|}{ LT (\%) } \\
\hline With & $0(0)$ & $21(4.3)$ & \multirow[t]{2}{*}{0.035} \\
\hline Without & $92(100)$ & 465 (95.7) & \\
\hline
\end{tabular}

Abbreviations: HT, Hashimoto's thyroiditis; LT, lymphocytic thyroiditis; PTC, papillary thyroid carcinoma; TNM, tumor-node-metastasis. 
Table 3 Univariate conditional logistic regression analysis of PTC patients

\begin{tabular}{|c|c|c|c|c|c|}
\hline & PTC n (\%) & BTD n (\%) & OR & $\mathrm{Cl}(5 \%-95 \%)$ & $P$-value \\
\hline \multicolumn{6}{|l|}{ Sex } \\
\hline Male & $92(15.9)$ & $89(14.4)$ & I & - & $0.45 I$ \\
\hline Female & $486(84.1)$ & $531(85.6)$ & 0.885 & $0.645,1.215$ & \\
\hline \multicolumn{6}{|l|}{ Age (years) } \\
\hline$<25$ & $46(8.0)$ & $17(2.7)$ & I & - & \\
\hline $25-44$ & $303(52.4)$ & $194(31.3)$ & 0.577 & $0.322,1.036$ & 0.065 \\
\hline $45-64$ & $200(34.6)$ & $327(52.7)$ & 0.226 & $0.126,0.405$ & $<0.001$ \\
\hline$\geq 65$ & $29(5.0)$ & $82(13.2)$ & 0.131 & $0.065,0.263$ & $<0.001$ \\
\hline \multicolumn{6}{|l|}{ Nodule size $(\mathrm{cm})$} \\
\hline$<1$ & $103(18.4)$ & $28(4.5)$ & 1 & - & \\
\hline $\mathrm{I}-$ & $204(36.4)$ & 140 (22.7) & 0.396 & $0.248,0.634$ & $<0.001$ \\
\hline $2-$ & $213(38.0)$ & $382(61.9)$ & 0.152 & $0.097,0.238$ & $<0.001$ \\
\hline $4-$ & $40(7.1)$ & $67(10.9)$ & 0.162 & $0.092,0.288$ & $<0.001$ \\
\hline \multicolumn{6}{|l|}{ TSH (mU/L) } \\
\hline$<0.3$ & $23(4.3)$ & $17(2.9)$ & 1.579 & $0.834,2.992$ & 0.161 \\
\hline $0.3-5.5$ & $472(88.2)$ & 55I (93.7) & 1 & - & \\
\hline$>5.5$ & $40(7.5)$ & $20(3.4)$ & 2.335 & $1.346,4.409$ & 0.003 \\
\hline \multicolumn{6}{|l|}{ TGAb (IU/mL) } \\
\hline$\leq 30$ & $376(80.9)$ & $523(94.6)$ & I & - & \\
\hline$>30$ & $89(19.1)$ & $30(5.4)$ & 4.127 & $2.672,6.372$ & $<0.001$ \\
\hline \multicolumn{6}{|l|}{ TPOAb (IU/mL) } \\
\hline$\leq 20$ & 351 (79.6) & $519(54.4)$ & 1 & - & \\
\hline$>20$ & $90(20.4)$ & $31(5.6)$ & 4.293 & $2.792,6.600$ & $<0.001$ \\
\hline \multicolumn{6}{|l|}{ TRAb (IU/mL) } \\
\hline$\leq 5$ & $261(69.0)$ & 465 (97.2) & I & - & \\
\hline$>5$ & $117(31.0)$ & $13(2.8)$ & 16.034 & $8.864,29.005$ & $<0.001$ \\
\hline \multicolumn{6}{|l|}{ TG $(\mu g / L)$} \\
\hline$\leq 20$ & 309 (84.9) & 348 (7I.9) & I & - & \\
\hline$>20$ & $55(15.1)$ & $136(28.1)$ & 0.455 & $0.321,0.645$ & $<0.001$ \\
\hline \multicolumn{6}{|l|}{ BMI $\left(\mathrm{kg} / \mathrm{m}^{2}\right)$} \\
\hline$<18.5$ & $33(9.6)$ & $37(6.6)$ & $\mathrm{I} .55 \mathrm{I}$ & $0.945,2.545$ & 0.083 \\
\hline $18.5-24.9$ & $24 I(70.1)$ & $419(74.8)$ & 1 & - & \\
\hline$\geq 25$ & $70(20.3)$ & $104(18.6)$ & 1.196 & $0.853,1.677$ & 0.368 \\
\hline \multicolumn{6}{|l|}{ Nodular goiter } \\
\hline Without & $500(45.5)$ & $600(3.2)$ & I & - & \\
\hline With & $78(47.3)$ & $20(96.8)$ & 4.68 & $2.823,7.758$ & $<0.001$ \\
\hline \multicolumn{6}{|l|}{ Follicular adenoma } \\
\hline Without & $470(8 \mathrm{I} .3)$ & $560(90.3)$ & I & - & \\
\hline With & $108(18.7)$ & $60(9.7)$ & 2.145 & $1.529,3.009$ & $<0.001$ \\
\hline \multicolumn{6}{|c|}{ Hashimoto's thyroiditis } \\
\hline Without & $508(87.9)$ & $585(94.4)$ & I & - & \\
\hline With & $70(12.1)$ & $35(5.6)$ & 2.303 & $1.509,3.515$ & $<0.001$ \\
\hline \multicolumn{6}{|c|}{ Lymphocytic thyroiditis } \\
\hline Without & $557(96.4)$ & $611(98.5)$ & I & - & \\
\hline With & $21(3.6)$ & $9(1.5)$ & 2.56 & $1.162,5.636$ & 0.02 \\
\hline \multicolumn{6}{|c|}{ Numbers of breeding babies $(n)$} \\
\hline$\leq 2$ & $397(51.0)$ & $382(49.0)$ & 1 & - & \\
\hline$\geq 3$ & $63(30.9)$ & $14 \mid(69.1)$ & 0.43 & $0.310,0.597$ & $<0.001$ \\
\hline \multicolumn{6}{|c|}{ Age of menarche (years) } \\
\hline$\leq 13$ & $201(54.8)$ & $166(45.2)$ & I & - & \\
\hline$>13$ & $256(42.3)$ & $349(57.7)$ & 0.606 & $0.467,0.787$ & $<0.001$ \\
\hline Menopause & & & & & \\
\hline Menstruating & $371(55.9)$ & $293(44.1)$ & I & - & \\
\hline Postmenopausal & $115(32.6)$ & $238(67.4)$ & 0.382 & $0.291,0.500$ & $<0.001$ \\
\hline Ethnicity & & & & & \\
\hline Han & $545(96.6)$ & $564(95.1)$ & I & - & \\
\hline Yi & $13(2.3)$ & $14(2.4)$ & 0.961 & $0.448,2.063$ & 0.919 \\
\hline Bai & $6(1.1)$ & $15(2.5)$ & 0.414 & $0.159,1.075$ & $0.4 \mid 4$ \\
\hline Residence & & & & & \\
\hline Urban areas & $419(72.5)$ & $453(73.1)$ & I & - & \\
\hline Rural areas & $159(27.5)$ & $167(26.9)$ & 1.029 & $0.7981,328$ & 0.824 \\
\hline
\end{tabular}

Abbreviations: BMI, body mass index; BTD, benign thyroid disease; $\mathrm{Cl}$, confidence interval; OR, odds ratio; PTC, papillary thyroid carcinoma; TG, thyroglobulin; TGAb, thyroglobulin antibody; TPOAb, thyroid peroxides antibody; TRAb, thyrotrophin receptor antibody; TSH, thyroid-stimulating hormone. 
with other types of benign tumor, the remaining PTC patients $(n=397)$ were divided into two groups - PTC combined with HT/LT (ie, PTC-HT/LT; n=90) and PTC alone $(n=307)$. There was no difference in the mean ages of the PTC-HT/ LT and PTC-only groups, which were 41.16 \pm 12.08 and $42.08 \pm 13.23$ years, respectively ( $t$-test, $P=0.92$ ). Mean tumor diameter was also similar between the two groups $(2.25 \pm 1.40 \mathrm{~cm}$ for PTC vs $1.99 \pm 1.24 \mathrm{~cm}$ for PTC-HT/LT). There were more TGAb- and TpoAb-positive patients in the PTC-HT/LT group ( $n=35$ or $46.7 \%$ and $n=16$ or $22.5 \%$, respectively) than in the PTC group $(P<0.001)$. There were 13 TRAb-positive patients (21\%) in the PTC-HT/LT group, which was fewer than that in the PTC group, although the difference was not statistically significant. The two groups were similar in terms of lymph node metastasis and TNM stage (Table 4). These data suggest that autoimmune thyroid diseases often occur in association with PTC.

\section{Female history \\ Menstrual history}

In the PTC group, 201 patients (54.8\%) experienced menarche at 13 years of age or younger, a number that was significantly higher than for the BTD group (166, 45.2\%) $(P<0.001)$. The number of menstruating and postmenopausal women also differed significantly between the two groups; there were fewer postmenopausal women in the PTC than in the BTD group $(32.6 \%$ vs $67.4 \%)(P<0.001)$ (Table 3$)$, indicating that early menarche and late menopause are linked to PTC occurrence.

Table 4 Clinicopathological features of PTC patients with or without HT/LT

\begin{tabular}{|c|c|c|c|}
\hline & $\begin{array}{l}\text { PTC without } \\
\text { HT/LT }\end{array}$ & $\begin{array}{l}\text { PTC with } \\
\text { HT/LT }\end{array}$ & $P$-value \\
\hline Nodule size $(\mathrm{cm})$ & $2.25 \pm 1.40$ & $1.99 \pm 1.24$ & 0.11 \\
\hline \multicolumn{4}{|c|}{ Lymphatic metastasis n (\%) } \\
\hline Yes & II $2(36.5)$ & 33 (36.7) & 0.97 \\
\hline No & $195(63.5)$ & $57(63.3)$ & \\
\hline \multicolumn{4}{|l|}{ TNM staging $\mathrm{n}(\%)$} \\
\hline I & $236(76.9)$ & $78(87.6)$ & 0.16 \\
\hline II & $18(5.9)$ & $2(2.2)$ & \\
\hline III & $20(6.5)$ & $3(3.4)$ & \\
\hline IV & $33(10.7)$ & $6(6.7)$ & \\
\hline \multicolumn{4}{|c|}{ TGAb (IU/mL) n (\%) } \\
\hline Positive & $36(14.8)$ & $35(46.7)$ & $<0.001$ \\
\hline \multicolumn{4}{|c|}{ TPOAb (IU/mL) n (\%) } \\
\hline Positive & $23(10.0)$ & $16(22.5)$ & $<0.001$ \\
\hline \multicolumn{4}{|c|}{ TRAb (IU/mL) n (\%) } \\
\hline Positive & $67(32.5)$ & $13(2 \mid .0)$ & 0.08 \\
\hline
\end{tabular}

Abbreviations: HT, Hashimoto's thyroiditis; LT, lymphocytic thyroiditis; PTC papillary thyroid carcinoma; TGAb, thyroglobulin antibody; TPOAb, thyroid peroxidase antibody; TRAb, thyrotrophin receptor antibody; TNM, tumor-node-metastasis.
Table 5 Multivariate conditional logistic regression analysis of PTC

\begin{tabular}{llll}
\hline Factors & P-value & OR & Cl (5\%-95\%) \\
\hline Age $(>45$ years) & $<0.00 I$ & 0.114 & $0.037-0.350$ \\
Nodule size $(>I \mathrm{~cm})$ & 0.003 & $0.37 \mathrm{I}$ & $0.194-0.708$ \\
$\mathrm{TG} \mu \mathrm{g} / \mathrm{L}$ & 0.010 & $0.48 \mathrm{I}$ & $0.275-0.840$ \\
$\mathrm{TGAb} \mathrm{IU} / \mathrm{mL}$ & $<0.00 \mathrm{I}$ & 4.894 & $2.520-9.505$ \\
$\mathrm{TRAb} \mathrm{IU} / \mathrm{mL}$ & $<0.00 \mathrm{I}$ & 16.047 & $4.047-63.628$ \\
\hline
\end{tabular}

Abbreviations: $\mathrm{Cl}$, confidence interval; OR, odds ratio; PTC, papillary thyroid carcinoma; TG, thyroglobulin; TGAb, thyroglobulin antibody; TRAb, thyrotrophin receptor antibody.

\section{Childbearing history}

The number of women who gave birth to $\leq 2$ children was similar between the PTC and BTD groups (51.0\% vs $49.0 \%$ ). However, the proportion PTC patients who gave birth to $\geq 3$ children was lower than that in the BTD group $(30.9 \%$ vs $69.1 \% ; P<0.001$ ) (Table 3), indicating that childbearing influences the incidence of PTC.

\section{Body mass index}

BMI information was obtained for 904 patients. According to WHO criteria, patients were divided into the following groups: underweight (BMI $<18.5 \mathrm{~kg} / \mathrm{m}^{2}$; $\mathrm{n}=70$ ), normal (18.5-24.9 kg/m²; $\mathrm{n}=660)$, and overweight $\left(\geq 25 \mathrm{~kg} / \mathrm{m}^{2}\right.$; $\mathrm{n}=174$ ). There were no differences in the incidence of benign and malignant thyroid nodules between weight categories ( $P=0.083$ and 0.368 for under- and overweight, respectively) (Table 3), demonstrating that the occurrence of PTC is unrelated to BMI.

\section{Multifactor analysis}

Benign and malignant thyroid nodules were the dependent variables, and age, nodule diameter, age at menarche, menopause, pregnancy, merged benign lesions, TSH level, TGAb, TRAb, TPOAb, and TG were the independent variables in the logistic regression analysis. The results showed that abnormal TGAb and TRAb were independent risk factors, whereas age $>45$ years, nodule diameter $>1 \mathrm{~cm}$, and TG were protective factors against PTC (Table 5).

\section{Discussion}

Thyroid cancer accounts for approximately $90 \%$ of cancers of the endocrine system, $1 \%$ of all malignancies, and $0.2 \%$ of malignancy-related mortality, globally, with a higher incidence among females than males. ${ }^{14}$ The etiology and pathogenesis of thyroid cancer are not clear. In this study, we investigated the clinical characteristics of PTC patients in the Yunnan plateau in order to identify risk factors for PTC in this geographical region.

TSH is a factor that induces the growth of the thyroid gland. Preoperative TSH level is positively correlated with 
thyroid malignancy, ${ }^{15}$ and TSH level is an independent risk factor for PTC. ${ }^{16}$ Serum TSH has also been shown to promote thyroid cancer cell growth, invasion, and angiogenesis. In the present study, TSH level was higher in PTC than in BTD patients, confirming the association between this hormone and the occurrence of PTC. However, we found that TSH was not an independent risk factor for PTC, which was in accordance with other reports. ${ }^{17,18}$ Another study has suggested that other growth factors such as insulin-like growth factor 1 play an important role in PTC pathogenesis. ${ }^{19}$

Autoimmune thyroid diseases, including GD and HT, are organ-specific and have an incidence of approximately $2 \%-5 \%$ in the general population. ${ }^{20-22}$ Chronic LT is the most common type of thyroid disease and is characterized by diffuse infiltration of lymphocytes into thyroid tissue, lymph follicle formation, and thyroid inflammation; a diagnosis of HT is made when these features are accompanied by eosinophilic changes in the follicular epithelium along with inflammatory cell infiltration into the thyroid gland. HT patients - predominantly women - also exhibited the blood autoantibodies TPOAb and TGAb. The coexistence of HT and PTC has led to the proposal that HT can evolve into thyroid cancer, ${ }^{23}$ although this is controversial. The incidence of concurrent PTC and HT is estimated to be $0.5 \%-35 \%,{ }^{24}$ while $28.2 \%$ of patients with HT develop PTC. ${ }^{25}$ In the Yunnan plateau, the incidence of PTC and concurrent HT or LT was $12.1 \%$ and $3.6 \%$, respectively; however, the presence of HT had no effect on tumor metastasis or invasion.

TGAb and TPOAb were detected at higher rates in PTC patients than in those with benign nodules, and a multivariate analysis showed that both are independent risk factors for PTC. The number of TGAb- and TPOAb-positive cases was higher in the PTC-HT/LT than in the PTC-only group; however, the TRAb-positive rate was lower in the PTC-HT/ LT group. Whether the presence of combined HT/LT can mitigate the risk of PTC due to reduced TRAb level is a question that will be addressed in future studies.

Overall, the present study analyses revealed that age $>45$ years, nodal size $>1 \mathrm{~cm}$, and elevated TG levels were protective factors in PTC. Abnormally elevated TGAb and TRAb levels were independent risk factors for PTC in females (Table 5).

TRAb is a diagnostic antibody for GD and had the highest risk coefficient for PTC among the factors examined. TRAb can have stimulatory (TSAb) or inhibitory (TSBAb) effects on the TSH receptor; the former can lead to follicular epithelial hyperplasia and hyperthyroidism, which can in turn result in PTC. On the other hand, TSAb binding can result in atrophic hypothyroidism, which can stimulate the secretion of TSH through a feedback mechanism, thereby promoting PTC. TRAb and TSH can act simultaneously to promote mitosis and block apoptosis of thyroid follicular cells, and TRAb stimulates thyroid cancer angiogenesis via activation of vascular endothelial and placental growth factors. ${ }^{26} \mathrm{We}$ therefore speculate that increased TRAb induces PTC. The incidence of thyroid nodules in the general population is approximately $5 \%$, while in GD patients the rate is as high as $10 \%-46 \%{ }^{27,28}$ A recent study found that in GD patients, the risk of breast and thyroid cancers was 1.58- and 10.4-fold higher, respectively, than in healthy individuals, especially in the 3 years preceding diagnosis, whereas the risk of developing PTC was 16-fold higher. ${ }^{29}$ The prognosis of patients with concurrent GD and PTC is also worse than those with either disease on their own. ${ }^{28,30}$ TRAb level is reportedly higher in patients with breast cancer and has been recommended as a breast cancer progression index; ${ }^{30}$ however, there have been no conclusive studies regarding the relationship between TRAb and PTC.

In developed countries, thyroid cancer is the second most prevalent type of solid tumor among women after breast cancer. ${ }^{31,32}$ In the general population, the incidence of PTC is approximately three times higher in women than in men, possibly due to the reproductive hormones and related factors. The highest rates of PTC are among women of childbearing age. ${ }^{33,34}$ Pregnancy, premature menopause, and gynecological diseases requiring estrogen replacement therapy can increase the risk of PTC. ${ }^{35}$ There is considerable variability in the findings of studies that have examined the association between PTC and hormones/reproductive risk factors. ${ }^{36}$ Estrogen and progesterone receptors are highly expressed in thyroid cancer patients, suggesting that PTC is a hormone-dependent tumor. ${ }^{37}$ Estrogen regulates the expression of a variety of target genes and promotes PTC cell proliferation, adhesion, invasion, and metastasis; it can also induce the formation of thyroid tumors, but then subsequently inhibits their growth and metastasis. ${ }^{38}$ This characteristic of estrogen may explain the high incidence but favorable outcome of thyroid cancer. The role of progesterone in thyroid cancer remains controversial; it is thought to increase thyroid cancer risk, ${ }^{39}$ but another study found that progesterone levels in PTC patients were lower than that in patients with benign tumors, ${ }^{40}$ suggesting that it has protective effects. In the present study, early menarche or late menopause was found to be associated with a higher incidence of PTC, and also an increased incidence of PTC was observed in women who had conceived two or fewer children, suggesting protective effect of progesterone against PTC. 
Epidemiological studies have shown that the risk of esophagus, colon, and kidney cancers, endometrial adenocarcinoma, and malignant melanoma are greater with increased BMI, suggesting that obesity precipitates the onset of cancer. ${ }^{41}$ The incidence of thyroid cancer was higher among adults who became overweight after childhood; ${ }^{42}$ one study found that a BMI $\geq 30$ was associated with larger tumor diameter, thyroid nodule invasion, and high TNM staging, but was unrelated to thyroid cancer recurrence. ${ }^{43}$ Others have reported that obesity is associated with an increased incidence but not invasiveness of thyroid cancer. ${ }^{44} \mathrm{We}$ did not find any relationship between BMI and PTC in the Yunnan plateau region, possibly due to large differences in weight between eastern and western populations.

In the present study, we reviewed the association between PTC risk factors and different altitudes or locations, which included inland or coastal areas in People's Republic of China (Table 6). Most research has focused on the plains region, and there is no report pertaining to the plateau area. A retrospective analysis ${ }^{45}$ of risk factors for PTC in Chinese cities of different geographical location and altitude revealed that in low-elevation coastal cities (ie, high-iodine areas such as Shanghai and Zhejiang Wenzhou), HT was an independent risk factor for PTC. In contrast, in low-altitude inland cities (eg, Beijing and Shandong Jinan), a high level of the autoantibody TGAb was an independent risk factor but HT was not. In high-altitude inland cities (ie, iodine-deficient areas such as Urumqi and Kunming in Yunnan province), high TGAb level was an independent risk factor. Our analysis also provides the first demonstration that TRAb is an independent risk factor for PTC in high-altitude, iodine-deficient areas. Whether the risk associated with high TRAb level is due to altitude, iodine deficiency, or both is uncertain. Serum TSH level was also increased in the PTC relative to the BTD group, but was not found to be an independent risk factor. Many studies of individuals in the plains have found that thyroid autoimmune diseases are linked to PTC; similarly, our study found that HT, while not an independent risk factor, frequently coexists with PTC.

The Yunnan plateau is located in southwestern China at an altitude of 1,500-2,500 $\mathrm{m}$ above sea level, occupying a unique geographical position. The mountainous area accounts for $84 \%-98 \%$ of the plateau. Iodine deficiency is widespread in Yunnan province, which comprises 16 prefectures (cities) and 129 counties (cities/districts) of $>45.7$ million inhabitants. In Yunnan, iodine content in water is low $(<150 \mu \mathrm{g} / \mathrm{L})^{46}$ and has been linked to the high incidence of thyroid cancer in this region.

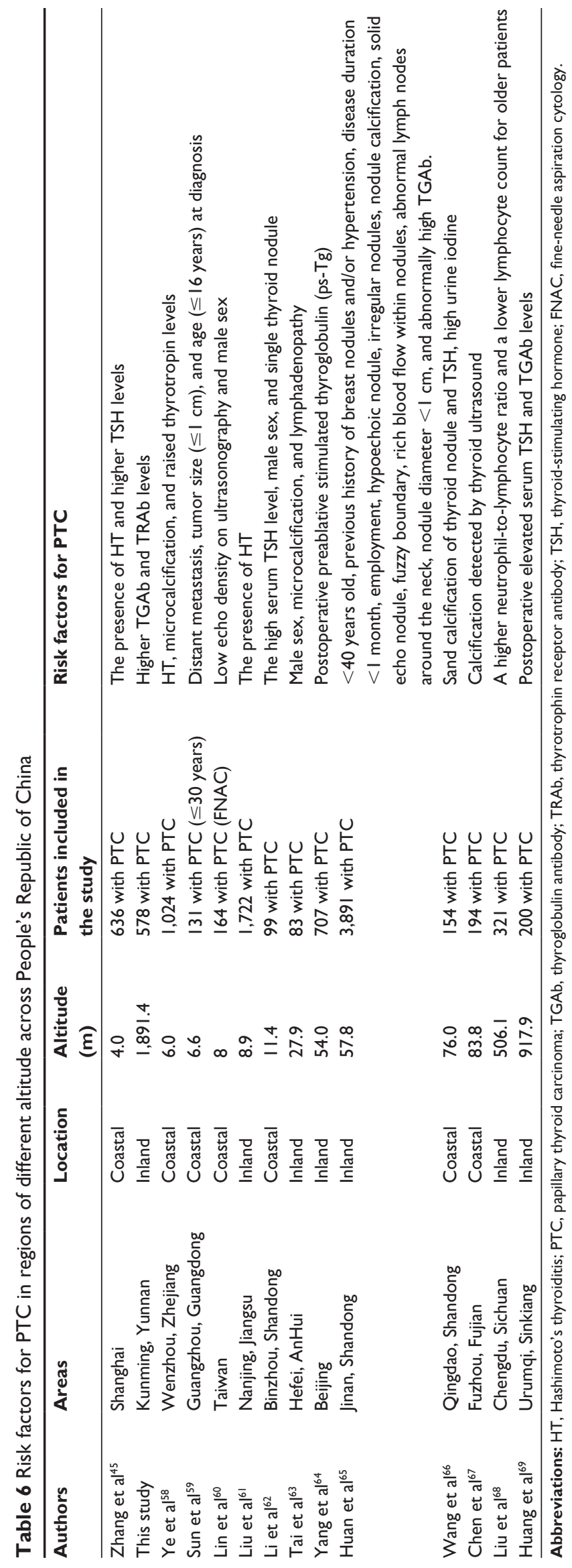


Universal salt iodization (USI) has been carried out throughout People's Republic of China since 1996, and iodine intake has consequently increased nationwide. This in conjunction with Yunnan iodine deficiency prevention measures has led to a significant improvement in iodine nutritional status among residents. However, iodine excess resulting from overexposure to environmental iodine in addition to poor monitoring is now more frequently observed than iodine deficiency and is a precipitating environmental factor in the development of autoimmune thyroid disease, hypo- and hyperthyroidism, and cancer. ${ }^{47-51}$ Overconsumption of iodized salt in food is associated with iodine excess and thyroid disorders in People's Republic of China. ${ }^{52-54}$

The prevalence of lymphocytic infiltration in non-neoplastic cases increases from $30 \%$ to $60.5 \%$ after salt iodization. ${ }^{55} \mathrm{~A}$ background of lymphocytic infiltration in neoplastic specimens also increased from $18.5 \%$ to $61 \%$ after iodine prophylaxis, whereas the frequency of papillary carcinoma in neoplastic specimens increased from $15 \%$ to $43 \%$. One study found that the incidence of TC was increased and the mean age of female patients with TC decreased after USI in the northeastern city of Shenyang, with PTC being the predominant histological type. ${ }^{11}$ There are a few possible mechanisms that can explain these effects, including the generation of reactive oxygen species, while oxidation and organification of excessive iodine in thyrocytes - likely due to a defect in the iodine processing machinery - can lead to increased oxidative stress and consequently, cell damage. ${ }^{55}$

A 2011 survey of iodine deficiency disorders monitoring in 30 counties in Yunnan province found that children's iodine nutrition status exceeded an appropriate level. ${ }^{46}$ Another study found that a large proportion of healthy children receiving iodine supplementation exhibited TRAb positivity without any predisposing conditions for GD. ${ }^{56}$ After nearly 20 years of iodine supplementation, in the past 10 years abnormally high levels of TGAb and TRAb, which are specific indicators of autoimmune thyroid disease, have been identified as independent risk factors for PTC in residents of Yunnan province. We therefore speculate that iodine supplementation has led to an increase in the prevalence of autoimmune thyroid disease in Yunnan, which has in turn caused an increase in the incidence of PTC.

Nonetheless, the PTC risk factors identified in our study did not differ from those reported in studies of populations living in the plains. The incidence of thyroid cancer has increased in all ethnic and racial groups over the past 2 decades; it is slightly lower among Hispanic people and Asians/Pacific islanders than among Caucasians. ${ }^{57}$ In addition to the Han majority, 26 ethnic groups live in this region, with some rural communities still practicing traditional agriculture and animal breeding. Owing to geographical characteristics of the plateau such as chronic hypoxia and distinctive cultural habits, economic development, urbanization, and disease status may vary considerably across the population. Despite the ethnic heterogeneity of Yunnan province, we did not note any ethnic differences in the occurrence of thyroid carcinoma.

\section{Conclusion}

Patient age, preoperative serum TSH level, autoimmune disease status, the presence of specific antibodies, and history of menstruation/fertility are linked to PTC incidence. HT was not an independent risk factor for, but was associated with, PTC. Elevated TRAb level is a risk factor for PTC in individuals living in the Yunnan plateau but not for those in the plains region. Although TRAb has not been reported as a risk factor for PTC in the plains, it was found to be a significant risk factor in the Yunnan plateau. The possibility that this is related to the altitude warrants further consideration. However, we do not argue against the salt iodization program, the benefits of which far outweigh any secondary effects.

\section{Acknowledgments}

This study was sponsored by grants from the Health Bureau of Yunnan Province (D-201203), the Science Technology Department of Yunnan Province (2013HB084), and the First People's Hospital of Yunnan Province Institute of Basic Medical Internal Institutions Project (2014N269).

\section{Disclosure}

The authors report no conflicts of interest in this work.

\section{References}

1. Vergamini LB, Frazier AL, Abrantes FL, Ribeiro KB, Rodriguez-Galindo C. Increase in the incidence of differentiated thyroid carcinoma in children, adolescents, and young adults: a population-based study. J Pediatr. 2014; 164:1481-1485.

2. Cramer JD, Fu P, Harth KC, Margevicius S, Wilhelm SM. Analysis of the rising incidence of thyroid cancer using the surveillance, epidemiology and end results national cancer data registry. Surgery. 2010;148: 1147-1152, discussion 52-53.

3. Colonna M, Bossard N, Guizard AV, Remontet L, Grosclaude P. Descriptive epidemiology of thyroid cancer in France: incidence, mortality and survival. Ann Endocrinol (Paris). 2010;71:95-101.

4. Mazeh H, Orlev A, Mizrahi I, Gross DJ, Freund HR. Concurrent medullary, papillary, and follicular thyroid carcinomas and simultaneous Cushing's syndrome. Eur Thyroid J. 2015;4:65-68.

5. Stansifer KJ, Guynan JF, Wachal BM, Smith RB. Modifiable risk factors and thyroid cancer. Otolaryngol Head Neck Surg. 2015;152: 432-437.

6. Qureshi IA, Khabaz MN, Baig M, Begum B, Abdelrehaman AS, Hussain MB. Histopathological findings in goiter: a review of 624 thyroidectomies. Neuro Endocrinol Lett. 2015;36:48-52. 
7. Liu YQ, Zhang SQ, Chen WQ, et al. [Trend of incidence and mortality on thyroid cancer in China during 2003-2007]. Zhonghua Liu Xing Bing Xиe Za Zhi. 2012;33:1044-1048. Chinese.

8. Qian BHM, Dong S, Wang J, Chen K. Incidence and mortality of thyroid cancers in Tianjin from 1981 to 2001. Chin J Endocinol Metab. 2005;21:432-434.

9. Yang L, Sun TT, Yuan YN, Wang N. [Time trends and pathological characteristics of thyroid cancer in urban Beijing, 1995-2010]. Zhonghua Yu Fang Yi Xue Za Zhi. 2013;47:109-112. Chinese.

10. Xie SH, Chen J, Zhang B, et al. Time trends and age-period-cohort analyses on incidence rates of thyroid cancer in Shanghai and Hong Kong. BMC Cancer. 2014;14:975.

11. Dong $\mathrm{W}$, Zhang $\mathrm{H}$, Zhang $\mathrm{P}$, et al. The changing incidence of thyroid carcinoma in Shenyang, China before and after universal salt iodization. Med Sci Monit. 2013;19:49-53.

12. Krain LS. Aviation, high altitude, cumulative radiation exposure and their associations with cancer medical hypotheses. Med Hypotheses. 1991;34:33-40.

13. Minelli G, Conti S, Manno V, Olivieri A, Ascoli V. The geographical pattern of thyroid cancer mortality between 1980 and 2009 in Italy. Thyroid. 2013;23:1609.

14. Jemal A, Murray T, Ward E, et al. Cancer statistics, 2005. CA Cancer J Clin. 2005;55:10-30.

15. Polyzos SA, Kita M, Efstathiadou Z, et al. Serum thyrotropin concentration as a biochemical predictor of thyroid malignancy in patients presenting with thyroid nodules. $J$ Cancer Res Clin Oncol. 2008;134: 953-960.

16. Haymart MR, Repplinger DJ, Leverson GE, et al. Higher serum thyroid stimulating hormone level in thyroid nodule patients is associated with greater risks of differentiated thyroid cancer and advanced tumor stage. J Clin Endocrinol Metab. 2008;93:809-814.

17. Matsuo K, Friedman E, Gejman PV, Fagin JA. The thyrotropin receptor (TSH-R) is not an oncogene for thyroid tumors: structural studies of the TSH-R and the alpha-subunit of Gs in human thyroid neoplasms. J Clin Endocrinol Metab. 1993;76:1446-1451.

18. Hurtado-Lopez LM, Monroy-Lozano BE, Martinez-Duncker C. TSH alone is not sufficient to exclude all patients with a functioning thyroid nodule from undergoing testing to exclude thyroid cancer. Eur J Nucl Med Mol Imaging. 2008;35:1173-1178.

19. Malaguarnera R, Morcavallo A, Belfiore A. The insulin and igf-I pathway in endocrine glands carcinogenesis. J Oncol. 2012;2012:635614

20. Stathatos N, Daniels GH. Autoimmune thyroid disease. Curr Opin Rheumatol. 2012;24:70-75.

21. Ban Y. Genetic factors of autoimmune thyroid diseases in Japanese Autoimmune Dis. 2012;2012:236981.

22. Jacobson EM, Tomer Y. The genetic basis of thyroid autoimmunity. Thyroid. 2007;17:949-961.

23. Dailey ME, Lindsay S, Skahen R. Relation of thyroid neoplasms to Hashimoto disease of the thyroid gland. AMA Arch Surg. 1955;70: 291-297.

24. Kim KW, Park YJ, Kim EH, et al. Elevated risk of papillary thyroid cancer in Korean patients with Hashimoto's thyroiditis. Head Neck. 2011;33:691-695.

25. Repplinger D, Bargren A, Zhang YW, Adler JT, Haymart M, Chen H Is Hashimoto's thyroiditis a risk factor for papillary thyroid cancer? J Surg Res. 2008;150:49-52.

26. Aydin F, Develi-Is S, Dogru-Abbasoglu S, et al. Polymorphisms of endothelin 1 (G5665T and T-1370G) and endothelin receptor type A $(\mathrm{C}+70 \mathrm{G}$ and G-231A) in Graves' disease. Int Immunopharmacol. 2014;18: 198-202.

27. Erbil Y, Barbaros U, Ozbey N, et al. Graves' disease, with and without nodules, and the risk of thyroid carcinoma. J Laryngol Otol. 2008;122: 291-295.

28. Pellegriti G, Mannarino C, Russo M, et al. Increased mortality in patients with differentiated thyroid cancer associated with Graves' disease. J Clin Endocrinol Metab. 2013;98:1014-1021.

29. Chen YK, Lin CL, Chang YJ, et al. Cancer risk in patients with Graves' disease: a nationwide cohort study. Thyroid. 2013;23:879-884.
30. Szychta P, Szychta W, Gesing A, Lewiński A, Karbownik-Lewińska M. TSH receptor antibodies have predictive value for breast cancer retrospective analysis. Thyroid Res. 2013;16:8.

31. Ferlay J, Soerjomataram I, Dikshit R, et al. Cancer incidence and mortality worldwide: sources, methods and major patterns in GLOBOCAN 2012. Int J Cancer. 2015;136:E359-E386.

32. Kilfoy BA, Zheng T, Holford TR, et al. International patterns and trends in thyroid cancer incidence, 1973-2002. Cancer Causes Control. 2009;20:525-531.

33. Dal Maso L, Bosetti C, La Vecchia C, Franceschi S. Risk factors for thyroid cancer: an epidemiological review focused on nutritional factors. Cancer Causes Control. 2009;20:75-86.

34. Brindel P, Doyon F, Rachedi F, et al. Menstrual and reproductive factors in the risk of differentiated thyroid carcinoma in native women in French Polynesia: a population-based case-control study. Am J Epidemiol. 2008; 167:219-229.

35. Zamora-Ros R, Rinaldi S, Biessy C, et al. Reproductive and menstrual factors and risk of differentiated thyroid carcinoma: the EPIC study Int $J$ Cancer. 2015;136:1218-1227.

36. Horn-Ross PL, Canchola AJ, Ma H, Reynolds P, Bernstein L. Hormonal factors and the risk of papillary thyroid cancer in the California Teachers Study cohort. Cancer Epidemiol Biomarkers Prev. 2011;20: 1751-1759.

37. Rajoria S, Hanly E, Nicolini A, et al. Interlinking of hypoxia and estrogen in thyroid cancer progression. Curr Med Chem. 2014;21:1351-1360.

38. Pályi I, Péter I, Daubner D, Vincze B, Lõrincz I. Establishment, characterization and drug sensitivity of a new anaplastic thyroid carcinoma cell line (BHT-101). Virchows Arch B Cell Pathol Incl Mol Pathol. 1993;63: 263-269.

39. Hannibal CG, Jensen A, Sharif H, Kjaer SK. Risk of thyroid cancer after exposure to fertility drugs: results from a large Danish cohort study. Hum Reprod. 2008;23:451-456.

40. Przybylik-Mazurek E, Hubalewska-Dydejczyk A, Fedorowicz A, Pach D. Factors connected with the female sex seem to play an important role in differentiated thyroid cancer. Gynecol Endocrinol. 2012;28: $150-155$.

41. Renehan G, Tyson M, Egger M, Heller RF, Zwahlen M. Body-mass index and incidence of cancer: a systematic review and meta-analysis of prospective observational studies. Lancet. 2008;371:569-578.

42. Kitahara M, Gamborg M, Berrington de Gonzalez A, Sorensen TI, Baker JL. Childhood height and body mass index were associated with risk of adult thyroid cancer in a large cohort study. Cancer Res. 2014;74:235-242.

43. Kim HJ, Kim NK, Choi JH, et al. Associations between body mass index and clinico-pathological characteristics of papillary thyroid cancer. Clin Endocrinol (Oxf). 2013;78:134-140.

44. Paes JE, Hua K, Nagy R, Kloos RT, Jarjoura D, Ringel MD. The relationship between body mass index and thyroid cancer pathology features and outcomes: a clinicopathological cohort study. J Clin Endocrinol Metab. 2010;95:4244-4250.

45. Zhang L, Li H, Ji QH, et al. The clinical features of papillary thyroid cancer in Hashimoto's thyroiditis patients from an area with a high prevalence of Hashimoto's disease. BMC Cancer. 2012;12:610.

46. Feng Y, Haitao Z, Hesong W, et al. Monitoring results of iodine deficiency disorders in Yunnan Province in 2011. Chin J Endemiol. 2013;32: 529-532.

47. Pearce EN, Andersson M, Zimmermann MB. Global iodine nutrition: where do we stand in 2013? Thyroid. 2013;23:523-528.

48. Zimmermann MB. Iodine deficiency and excess in children: worldwide status in 2013. Endocr Pract. 2013;19:839-846.

49. Zimmermann MB, Andersson M. Update on iodine status worldwide. Curr Opin Endocrinol Diabetes Obes. 2012;19:382-387.

50. Camargo RY, Tomimori EK, Neves SC, et al. Thyroid and the environment: exposure to excessive nutritional iodine increases the prevalence of thyroid disorders in Sao Paulo, Brazil. Eur J Endocrinol. 2008;159:293-299.

51. Laurberg P, Cerqueira C, Ovesen L, et al. Iodine intake as a determinant of thyroid disorders in populations. Best Pract Res Clin Endocrinol Metab. 2010;24:13-27. 
52. Li S, Zheng Q, Xu J, Gorstein J, Wang H, Dong H. Iodine excess or not: analysis on the necessity of reducing the iodine content in edible salt based on the national monitoring results. Asia Pac J Clin Nutr. 2011;20: 501-506.

53. Li S, Fan Y, Chen H, et al. Is the current iodine content in edible salt appropriate for eliminating iodine deficiency in China. Asia Pac J Clin Nutr. 2010;19:231-235.

54. Teng X, Shan Z, Chen Y, et al. More than adequate iodine intake may increase subclinical hypothyroidism and autoimmune thyroiditis: a cross-sectional study based on two Chinese communities with different iodine intake levels. Eur J Endocrinol. 2011;164:943-950.

55. Luo Y, Kawashima A, Ishido Y, et al. Iodine excess as an environmental risk factor for autoimmune thyroid disease. Int J Mol Sci. 2014;15: 12895-12912.

56. Okten A, Akcay S, Cakir M, Girisken I, Kosucu P, Deger O. Iodine status, thyroid function, thyroid volume and thyroid autoimmunity in patients with type 1 diabetes mellitus in an iodine-replete area. Diabetes Metab. 2006;32:323-329.

57. Magreni A, Bann DV, Schubart JR, Goldenberg D. The effects of race and ethnicity on thyroid cancer incidence. JAMA Otolaryngol Head Neck Surg. 2015;141:319-323.

58. Ye ZQ, Gu DN, Hu HY, Zhou YL, Hu XQ, Zhang XH. Hashimoto's thyroiditis, microcalcification and raised thyrotropin levels within normal range are associated with thyroid cancer. World J Surg Oncol. 2013;11:56.

59. Sun CZ, Chen FJ, Zeng ZY, et al. [Differentiated thyroid carcinoma in young people]. Zhonghua Er Bi Yan Hou Tou Jing Wai ke Za Zhi. 2005;40:595-600. Chinese.

60. Lin JD, Hsueh C, Chao TC, Weng HF, Huang BY. Thyroid follicular neoplasms diagnosed by high-resolution ultrasonography with fine needle aspiration cytology. Acta Cytol. 1997;41:687-691.
61. Liu X, Zhu L, Cui D, et al. Coexistence of histologically confirmed Hashimoto's thyroiditis with different stages of papillary thyroid carcinoma in a consecutive Chinese cohort. Int J Endocrinol. 2014;2014:769294.

62. Li JZ, Jin YJ, Liu X, Zhang LY. [Association between the serum TSH concentration and thyroid cancer incidence]. Zhonghua Zhong Liu Za Zhi. 2011;33:921-924. Chinese.

63. Tai JD, Yang JL, Wu SC, Wang BW, Chang CJ. Risk factors for malignancy in patients with solitary thyroid nodules and their impact on the management. J Cancer Res Ther. 2012;8:379-383.

64. Yang X, Liang J, Li TJ, et al. Postoperative stimulated thyroglobulin level and recurrence risk stratification in differentiated thyroid cancer. Chin Med J (Engl). 2015;128:1058-1064.

65. Huan Q, Wang K, Lou F, et al. Epidemiological characteristics of thyroid nodules and risk factors for malignant nodules: a retrospective study from 6,304 surgical cases. Chin Med J (Engl). 2014;127:2286-2292.

66. Wang F, Wang Y, Wang L, et al. Strong association of high urinary iodine with thyroid nodule and papillary thyroid cancer. Tumour Biol. 2014;35:11375-11379.

67. Chen G, Zhu XQ, Zou X, et al. Retrospective analysis of thyroid nodules by clinical and pathological characteristics, and ultrasonographically detected calcification correlated to thyroid carcinoma in South China. Eur Surg Res. 2009;42:137-142.

68. Liu J, Du J, Fan J, et al. The neutrophil-to-lymphocyte ratio correlates with age in patients with papillary thyroid carcinoma. ORL J Otorhinolaryngol Relat Spec. 2015;77:109-116.

69. Huang T, Li W-X, Zhang L. Relationship between differentiated thyroid cancer and serum thyroid stimulating hormone autoantibodies. Chin Gen Pract. 2013;16:4258-4260.
Therapeutics and Clinical Risk Management

\section{Publish your work in this journal}

Therapeutics and Clinical Risk Management is an international, peerreviewed journal of clinical therapeutics and risk management, focusing on concise rapid reporting of clinical studies in all therapeutic areas, outcomes, safety, and programs for the effective, safe, and sustained use of medicines. This journal is indexed on PubMed Central, CAS,

\section{Dovepress}

EMBase, Scopus and the Elsevier Bibliographic databases. The manuscript management system is completely online and includes a very quick and fair peer-review system, which is all easy to use. Visit $\mathrm{http}: / / \mathrm{www}$.dovepress.com/testimonials.php to read real quotes from published authors. 